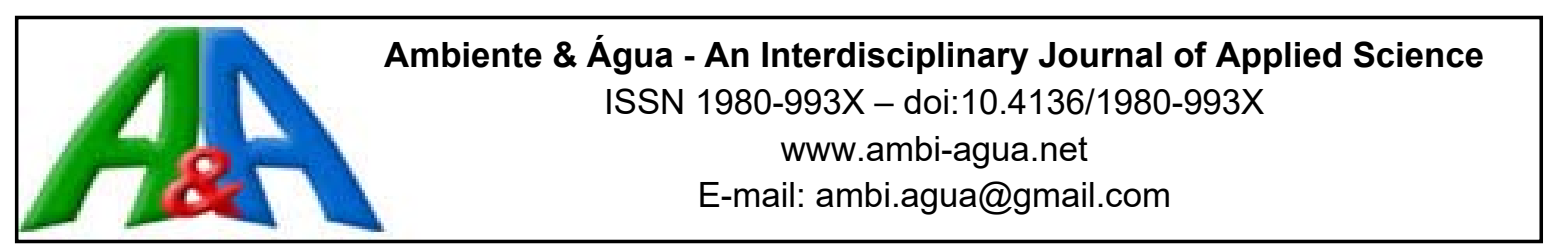

\title{
Variabilidade de concentração de metano na região do Pantanal Mato- grossense usando dados do satélite AQUA
}

\author{
doi:10.4136/ambi-agua.1985
}

Received: 16 Aug. 2016; Accepted: 03 Feb. 2017

\author{
Tonny Jader de Moraes*; Fernando da Silva Sallo; Carlo Ralph de Musis; \\ Luciana Sanches; Iramaia Jorge Cabral de Paulo; Rafael da Silva Palácios
Universidade Federal de Mato Grosso (UFMT) / Instituto de Física (IF), Cuiabá, MT, Brasil
Programa de Pós-Graduação em Física Ambiental
*Autor correspondente: e-mail: tonny@fisica.ufmt.br, fdss88@gmail.com, carlo.demusis@gmail.com, lsanches@hotmail.com, iramaiaj@gmail.com, rafael.pgfa@gmail.com

\section{RESUMO}

A emissão de metano é um fator importante na gestão de áreas alagadas como o Pantanal. Zonas úmidas em todo o mundo contribuem para o ciclo global do $\mathrm{CH}_{4}$, emitindo cerca de um terço do $\mathrm{CH}_{4}$ global. No entanto, a dinâmica de $\mathrm{CH}_{4}$ na atmosfera acima do Pantanal, continua mal compreendida. Neste trabalho estudou-se a variabilidade de $\mathrm{CH}_{4}$ na atmosfera na região do Pantanal com base na série temporal de concentração de $\mathrm{CH}_{4}$ (2003-2013), a partir de dados do sensor AIRS a bordo do satélite AQUA, e se analisou sua relação com a precipitação. A metodologia utilizada baseou-se em estatísticas descritivas que incluem médias semanais, anomalias baseadas na variância, correlação cruzada entre precipitação e concentração de $\mathrm{CH}_{4}$, e transformada wavelet. Os resultados mostraram que existe uma concentração média sazonal e a variabilidade da anormalidade de $\mathrm{CH}_{4}$ com uma precipitação é negativa. Variações sub-anuais de $\mathrm{CH}_{4}$ foram inversamente proporcionais às de precipitação, devido possivelmente ao aumento da concentração de hidroxila [OH-] durante a estação chuvosa, o que diminui o saldo de emissão de $\mathrm{CH}_{4}$. A análise wavelet permitiu observar um ciclo anual e interanual da concentração de $\mathrm{CH}_{4}$ entre 2009 a 2013.

Palavras-chave: correlação cruzada, precipitação, wavelet.

\section{Methane concentration variability in Pantanal region in the Mato Grosso State using data from AQUA satellite}

\begin{abstract}
Methane emission is an important factor in the management of wet areas such as the Pantanal region. The temporal variability of $\mathrm{CH}_{4}$ concentrations on the Pantanal was therefore studied using remote sensing data from 2003 to 2013 from an AIRS sensor on board of AQUA satellite. Wetlands worldwide contribute to the overall $\mathrm{CH}_{4}$ cycle, emitting around one-third of the overall $\mathrm{CH}_{4}$. However, the dynamics of $\mathrm{CH}_{4}$ remain poorly understood in the Pantanal. This work studied the variability of $\mathrm{CH}_{4}$ in the atmosphere over the Pantanal of Mato Grosso, based on the time series of $\mathrm{CH}_{4}$ concentration (2002-2013). The methodology used was based on descriptive statistics that included weekly averages, variance anomalies, cross-correlation between precipitation and concentration of $\mathrm{CH}_{4}$, and the transformed wavelet technique. The
\end{abstract}


results showed a seasonal average concentration and the variability of $\mathrm{CH}_{4}$ abnormality in a negative cross-correlation with precipitation. Sub-annual variations of $\mathrm{CH}_{4}$ were inversely related to precipitation, and seemed to be influenced by the concentration of hydroxyl [OH-] during the rainy season, which reduces the balance of $\mathrm{CH}_{4}$ emissions. The wavelet analysis found an annual and inter-annual cycle of $\mathrm{CH}_{4}$ concentration between 2009-2013.

Keywords: cross-correlation, rainfall, wavelet.

\section{INTRODUÇÃO}

$\mathrm{O}$ metano $\left(\mathrm{CH}_{4}\right)$ é o composto químico orgânico mais abundante da atmosfera. Vários estudos indicam que sua concentração vem aumentando de aproximadamente 722 ppb em 1750, para $1802 \pm 1 \mathrm{ppb}$ (Dlugokencky et al., 2011) atingindo mais que o dobro desde o período pré-industrial (Bousquet et al., 2006). As áreas alagáveis são importantes por serem sensíveis as modificações climáticas e são os maiores emissores naturais de $\mathrm{CH}_{4}$, sendo que o seu aumento pode atuar como uma retroalimentação positiva para o aquecimento futuro (Melton et al., 2013).

As áreas alagáveis são a maior fonte natural de $\mathrm{CH}_{4}$ na atmosfera, contribuindo $145 \mathrm{Tg}$ ano $^{-1}$, o que corresponde a cerca de $24 \%$ da emissão total de todas as fontes, e são especialmente importantes durante o período das chuvas (Bousquet et al., 2011). No Brasil há um alagamento sazonal em grandes áreas como a região Amazônica e o Pantanal. A inundação sazonal do Pantanal traz um conjunto de características distintas em suas dinâmicas de nutrientes e pulso de inundação (Vourlitis et al., 2013). Nessa área a vazão de água é reduzida, formando um ambiente favorável à deposição e posterior degradação da matéria orgânica de forma aeróbica e anaeróbica, resultando na formação de gases $\mathrm{CO}_{2}$ e $\mathrm{CH}_{4}$.

Uma das alternativas para construção de modelos para estimativa das emissões de $\mathrm{CH}_{4}$ é via uso de informações obtidas por sensoriamento remoto, viabilizando estudo em locais com poucas informações coletadas in loco. Por exemplo, estudos demonstraram a viabilidade e a vantagem de utilizar o sensor Atmospheric Infrared Sounder (AIRS) a bordo da plataforma AQUA, para inferir e monitorizar a concentração de gases na atmosfera, oferecendo uma grande oportunidade para coletar e inferir a concentração de alguns gases-traço de efeito de estufa na atmosfera da Terra (Xiong et al., 2013; Costa et al., 2013).

$\mathrm{O}$ objetivo deste trabalho foi quantificar a concentração de $\mathrm{CH}_{4}$ no Pantanal Mato-grossense, analisar a variabilidade temporal da concentração de metano na atmosfera em função da variabilidade de precipitação pluviométrica por meio de correlação cruzada, bem como avaliar a dinâmica da concentração do gás $\mathrm{CH}_{4}$ por meio da análise wavelet (ou ondoleta) com base na série temporal de 2002-2013.

\section{MATERIAL E MÉTODO}

Este estudo foi realizado em uma área no Norte do Pantanal Mato-grossense compreendida

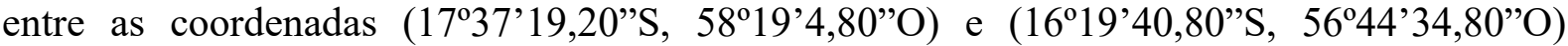
(Figura 1), altitude entre 80 a $120 \mathrm{~m}$. A região está sob domínio climático Aw, segundo a classificação de Köppen, com duas estações bem definidas, uma seca, de abril a setembro e outra úmida, de outubro a março, com precipitação média anual superior a $1300 \mathrm{~mm}$.

Os dados utilizados de $\mathrm{CH}_{4}$ foram estimados pelos sensores AIRS a bordo do satélite AQUA, no período 2002-2013, com concentrações médias diárias de taxa de mistura de $\mathrm{CH}_{4}$ (VMR, volume mixing ratio) para três níveis de pressão atmosférica $(160,5 ; 260 ; 359 \mathrm{hPa})$. Os dados AIRS são distribuídos pela NASA Goddard Earth Sciences Data Information and Services Center (DISC). A resolução espacial é $1^{\circ}$ por $1^{\circ}$ de latitude e longitude, com

Rev. Ambient. Água vol. 12 n. 3 Taubaté - May / Jun. 2017 
periodicidade diária e cobertura global. Além disso, foram utilizados dados de precipitação média diária ( $\mathrm{mm} \mathrm{dia}^{-1}$ ) obtidos pelo produto TRMM (Daily TRMM and Other Rainfall Estimate) no período de 2002-2015.

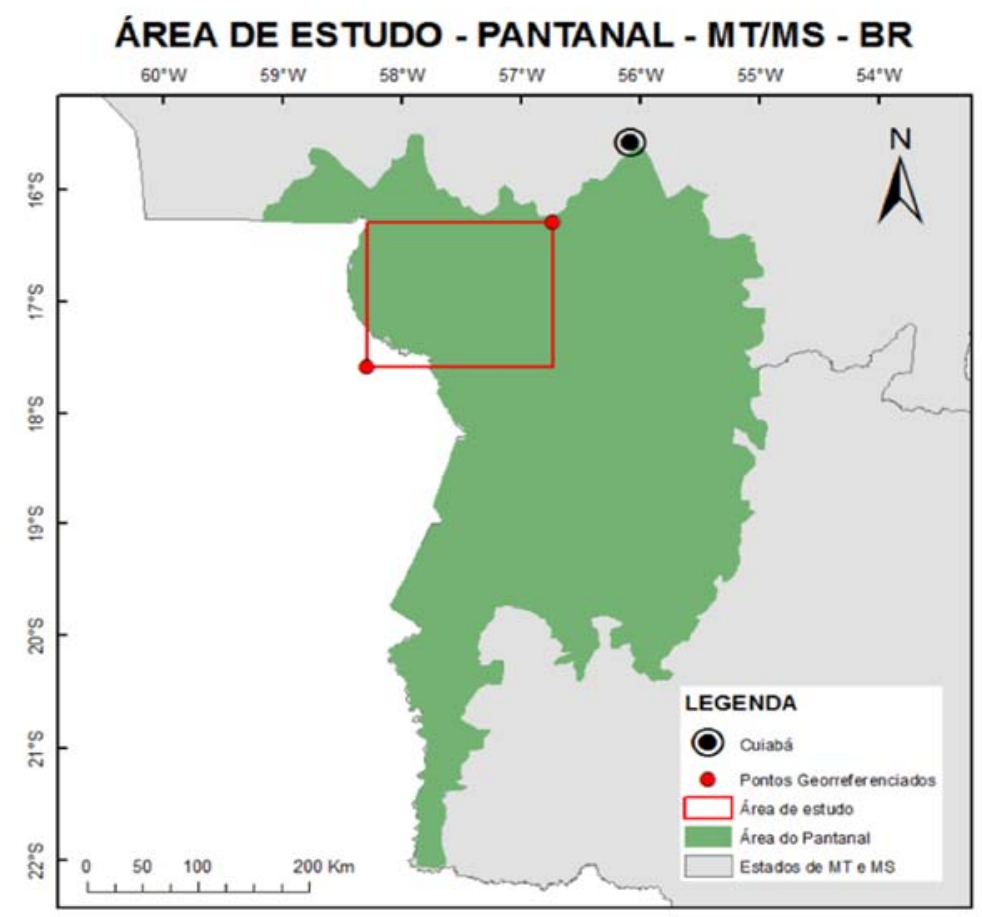

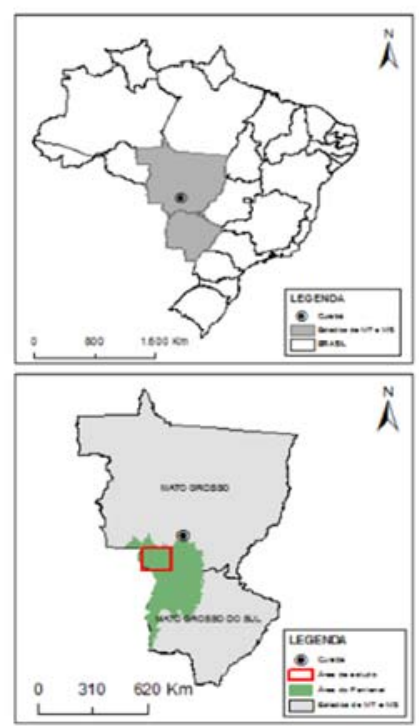

Sistema de Coordenada Geográica: SIRGAS 2000 Elaborado por: Karen F. A. P. Sallo

Figura 1. Localização da área de estudo georreferenciada e delimitada pelos pontos e pela linha em vermelho, aproximadamente $60 \mathrm{~km}$ da cidade de Cuiabá, Mato Grosso, Brasil. A área de estudo abrange pouco mais de 2 pixels.

As investigações sobre a modulação de taxa de mistura de $\mathrm{CH}_{4}$ no Pantanal foi realizada inicialmente pelo cálculo das médias de 8 dias e desvio padrão (DP) da concentração de $\mathrm{CH}_{4}$ nos três perfis de pressão atmosféricas. Esse procedimento propiciou um exploratório para variabilidade temporal. Em seguida foi removida a variabilidade anual da série para os três níveis de pressão através do cálculo da anomalia e da anomalia acumulada, com objetivo de analisar a variabilidade interanual da concentração de $\mathrm{CH}_{4}$ no período 2002-2013.

As variações temporais da concentração de $\mathrm{CH}_{4}$ foram avaliadas por meio da análise de ondoleta, que possibilitam a decomposição da série como uma função de tempo e da frequência. A ondoleta Morlet é uma função do tempo t, composta por uma exponencial complexa e modulada por uma exponencial gaussiana (Torrence e Compo, 1997). A função é da família ondoletas complexas não ortonormais dada pela Equação 1.

$$
\psi(t)=\pi^{-1 / 4} \mathrm{e}^{\mathrm{iw_{0 }} \mathrm{t}} \mathrm{e}^{-1 / 2 \mathrm{t}^{2}}, \text { para } \mathrm{w}_{0} \geq 5
$$

em que:

$\Psi$ é o valor ondoleta para um parâmetro adimensional t,

wo a frequência adimensional. Admite-se w0 $=6$ para satisfazer a condição de admissibilidade; deste modo a função deve ter média zero e ser localizada tanto no espaço tempo como na frequência (Torrence e Compo, 1997).

A ondoleta foi utilizada pelos autores Torrence e Compo (1997) e Beecham e Chowdbury (2010) na análise de séries temporais de precipitação. A técnica foi utilizada na análise de fluxo e concentração de $\mathrm{CH}_{4}$ na atmosfera por Koebsch et al. (2015) e Costa et al. (2013). Neste 
estudo, a análise de ondoleta foi aplicada utilizando-se o algoritmo elaborado por Torrence e Compo (1997).

Para as análises da série temporal de precipitação efetuou-se a média, anomalia e anomalia acumulada, usando como base o tempo mensal do período 2002-2013. Essa análise exploratória possibilitou observar padrões não-sazonais e variações ao longo da série. Posteriormente, realizou-se a análise de correlação cruzada em escala semanal para as séries de anomalia de chuva e anomalia de concentração de $\mathrm{CH}_{4}$ para o mesmo período de dados da precipitação 2002-2013.

\section{RESULTADO E DISCUSSÃO}

Observa-se uma relação entre as séries da concentração de $\mathrm{CH}_{4}$ e a precipitação, sendo possível observar máximos coincidentes (Figura 2). Detectou-se sazonalidade nas concentrações de $\mathrm{CH}_{4}$ nos níveis de pressão (Figura 2), com as maiores concentrações no nível de 359 mb com média ( \pm DP) $1766 \pm 10$ ppbv. Ao nível de pressão de $160 \mathrm{mb}$, a média $( \pm \mathrm{DP})$ da concentração foi $1728 \pm 17$ ppbv, apresentando maior amplitude entre as concentrações máximas e mínimas. Também, é mais aparente a sazonalidade da concentração do $\mathrm{CH}_{4}$ em nível de pressão $160 \mathrm{mb}$, com uma média $( \pm \mathrm{DP})$ de $1728 \pm 17 \mathrm{ppbv}$, porém menos evidente em 359 $\mathrm{mb}$. Esse resultado foi semelhante aos trabalhos de Costa et al. (2013) e Ribeiro et al. (2016), que trabalharam com dados de sensoriamento relativos a áreas alagadas no Estado do Amazonas. Do mesmo modo, houve uma diminuição da concentração de $\mathrm{CH}_{4}$ em função da diminuição dos níveis de pressão, sendo justificada pelos principais processos de remoção de $\mathrm{CH}_{4}$ da atmosfera, que são a reação de $\mathrm{CH}_{4}$ com radicais hidroxila $\left(\mathrm{OH}^{-}\right)$na troposfera e estratosfera e com átomos de oxigênio na estratosfera (Dlugokencky et al., 2011). Resultados semelhantes também foram obtidos por Alvalá et al. (2004), que analisaram a concentração de $\mathrm{CH}_{4}$ na região do Atlântico Sul com medidas realizadas a $25 \mathrm{~km}$ da costa brasileira, utilizando a método trajetórias isentrópicas, observando uma variação sazonal com uma média $( \pm \mathrm{DP})$ de $1707 \pm 8$ ppbv.

Durante a estação seca (maio a setembro), verificou-se a média ( \pm DP) das concentrações de $\mathrm{CH}_{4}$ de $1736 \pm 16 ; 1754 \pm 10$ e $1765 \pm 11$ ppbv nos níveis pressão 160,5; 260 e $359 \mathrm{mb}$, respectivamente. Enquanto que durante a estação chuvosa (janeiro a abril) as médias $( \pm \mathrm{DP})$ foram $1720 \pm 14 ; 1746 \pm 11$ e $1751 \pm 16$ ppbv em nível de pressão 160,5; 260 e 359 mb, respectivamente. As maiores concentrações de $\mathrm{CH}_{4}$ durante a estação seca, de acordo com Spivakovsky et al. (1990) e McNorton et al. (2016), podem ser explicadas pela sazonalidade do radical $\mathrm{OH}^{-}$na troposfera e estratosfera. Ainda Spivakovsky et al. (1990) sugerem que, na latitude próxima à região de estudo, a concentração de $\mathrm{OH}^{-}$é maior no período chuvoso, servindo como sumidouro de $\mathrm{CH}_{4}$. Conforme observado por Marani e Alvala (2006), a variação de fluxo de $\mathrm{CH}_{4}$ está associada à variação anual de área alagada no pantanal, que por sua vez está relacionada a sazonalidade de precipitação. De acordo com os resultados de Ribeiro et al., (2016), ocorre um pico de precipitação seis meses antes do maior pico de concentração de $\mathrm{CH}_{4}$, na região da Amazônia. E ainda Bastviken et al. (2010), sugerem que o metabolismo de matéria orgânica está relacionado à estação hidrológica - com a crescente das águas no Pantanal, ocorre a maior taxa de metabolismo de matéria orgânica.

É possível observar um padrão das anomalias ao longo de toda a série, entretanto foi notado um aumento das anomalias positivas a partir de $2007 \mathrm{e}$, posteriormente, aumento das anomalias negativas após 2011 (Figura 3A). Este resultado pode ser verificado (Figura 3A) pelas séries de anomalia acumulada, onde é possível observar uma tendência de diminuição da precipitação nos primeiros 4 anos da série e, posteriormente, aumento das anomalias positivas em 2007, e após 2011 apareceu aumento de anomalias negativas. Esse resultado foi consistente com o de

Rev. Ambient. Água vol. 12 n. 3 Taubaté - May / Jun. 2017 
Penatti et al. (2015), que verificaram, por sensoriamento remoto, mudanças espaço-temporais das variáveis do balanço hídrico no Pantanal. Anomalias positivas de estoque de água terrestre medidos foram maiores nos anos de 2006, 2007, 2008 e 2011, com menor pico ocorrido em 2012. Hamilton et al. (2002) destacaram que a maior parte da variabilidade climática observada é conduzida pelas anomalias na temperatura superficial das águas do Oceano Pacífico, com os fenômenos El Niño e La Niña influenciando diretamente na taxa de precipitação na bacia pantaneira e seus afluentes. Nesse contexto, um fator importante na emissão de $\mathrm{CH}_{4}$ no Pantanal para a atmosfera é a extensão espacial da área alagada. Essa extensão é primariamente controlada pela variação sazonal do nível dos rios e pelo acúmulo de água da precipitação. Com base em dados de satélite, Hamilton et al. (2002) obtiveram estimativas sobre o alagamento no Pantanal, os quais foram utilizados para estimativas de emissão de $\mathrm{CH}_{4}$ do Pantanal.

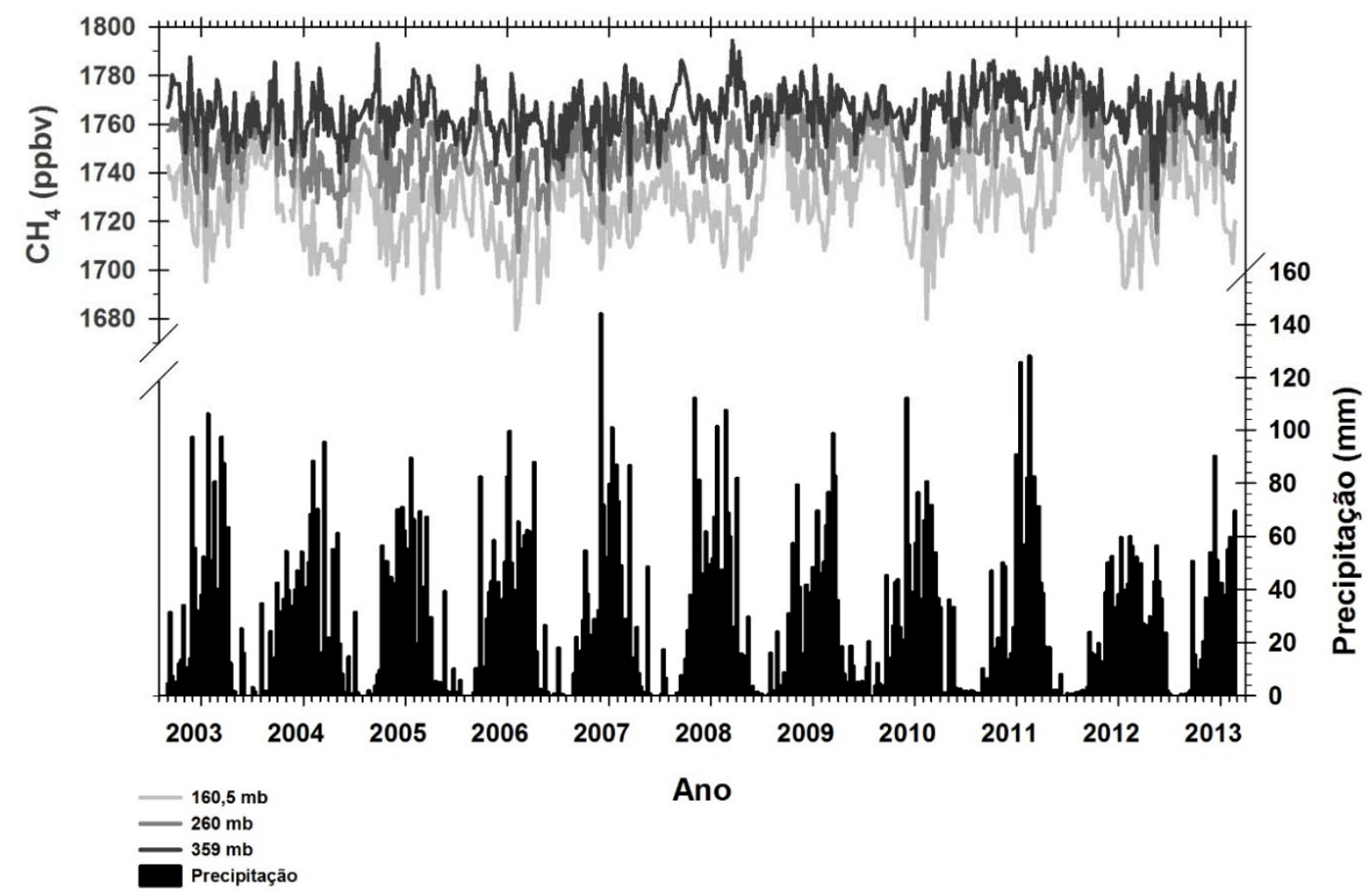

Figura 2. Série temporal de concentração média de 8 dias de $\mathrm{CH}_{4}$, e de precipitação sobre a região do Pantanal Mato-grossense, de 2002 a 2013. As linhas em tom de cinza (acima) com escala a esquerda em três níveis de pressão atmosférica, iniciando mais acima na escala $359 \mathrm{mb}, 260 \mathrm{mb}$ e 160,5 mb, terminando mais abaixo na escala, respectivamente. Na parte inferior da figura em preto com escala à direita, observa-se a série de precipitação.

Nas análises da anomalia da concentração de $\mathrm{CH}_{4}$ (Figura 3) observa-se que em geral houve aumento das anomalias positivas de concentração de $\mathrm{CH}_{4}$ a partir de $2007 \mathrm{em}$ todos os níveis de pressão atmosférica, sendo menos evidente no nível $359 \mathrm{mb}$ (Figura 3C). Nota-se também a predominância de negativos das anomalias entre os anos de 2003 a 2007. Contudo, a maior variabilidade da anomalia ocorreu no nível de pressão 160 mb (Figura 3A), entre 49,6 a $-52,6$ ppbv. A tendência da dinâmica de concentração de $\mathrm{CH}_{4}$ pode ser observada na série de anomalia acumulada (Figura 3) pelo decréscimo da concentração de $\mathrm{CH}_{4}$ para 2002-2006, e aumento em 2007 a 2013, nos níveis de pressão analisados. 
$\mathrm{Na}$ anomalia acumulada da precipitação (Figura 3D) observa-se dois comportamentos que governam as oscilações das anomalias de $\mathrm{CH}_{4}$. $\mathrm{O}$ primeiro refere-se às variações sub-anuais de $\mathrm{CH}_{4}$, que é inversamente proporcional ao ciclo anual de precipitação. O segundo refere-se ao aumento da concentração de $\mathrm{CH}_{4}$ a partir de 2008, que tem origem do aumento progressivo do alagamento a partir de 2006 até 2012. O aumento de alagamento estar relacionado as variações interanuais de precipitação, que por sua vez estão relacionadas com os fenômenos de El Niño e La Niña (Ribeiro et al., 2016).

A
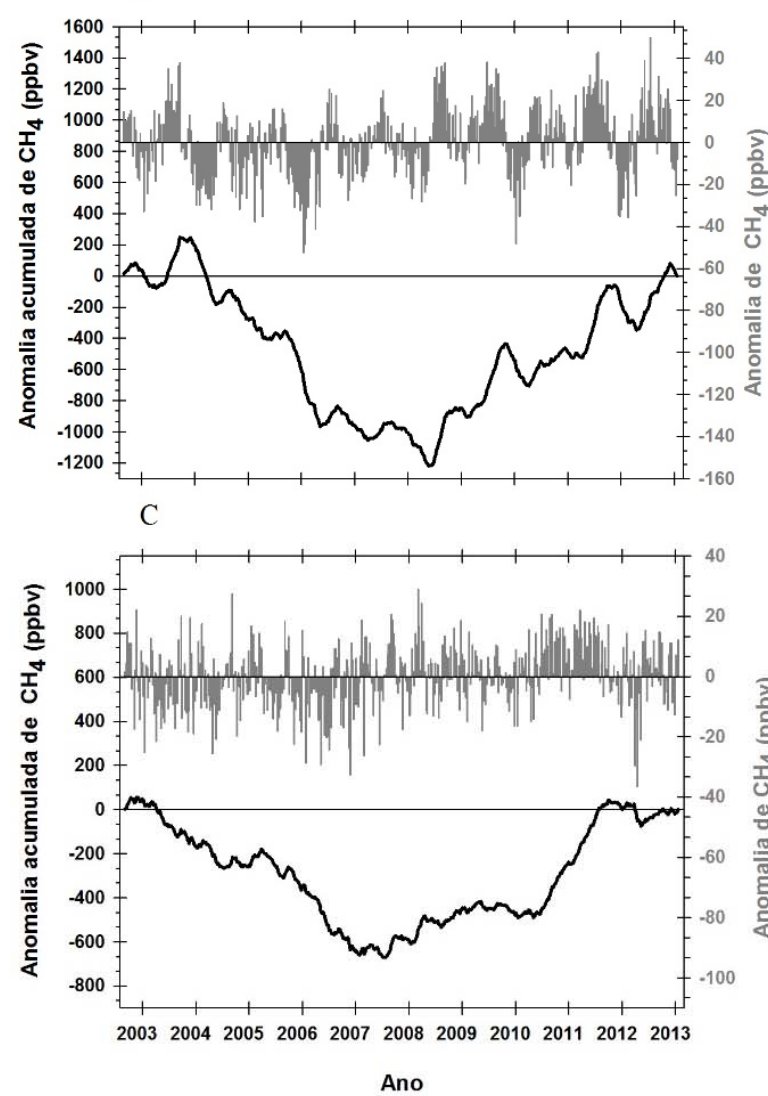

B

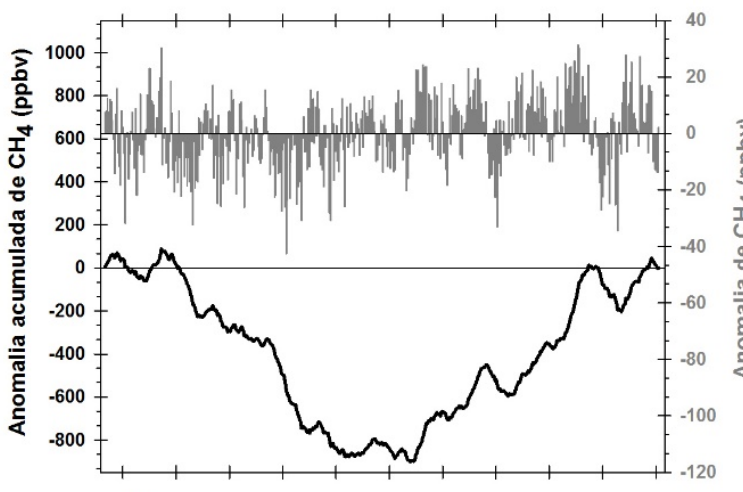

D

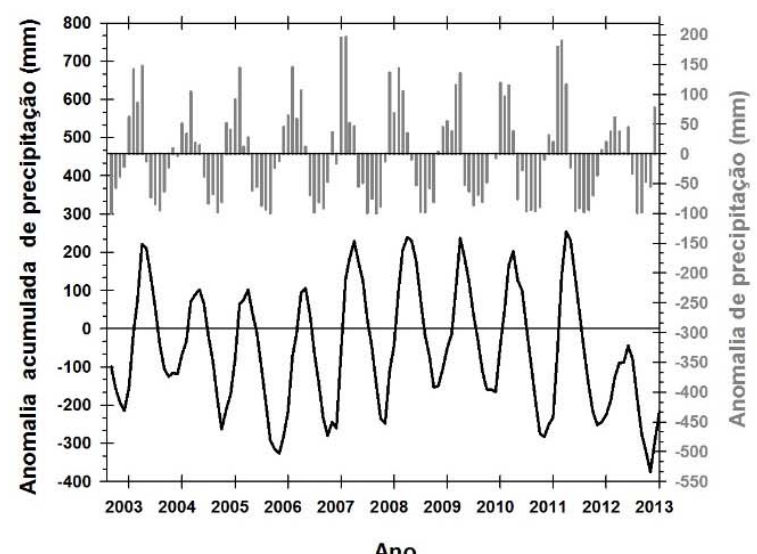

Ano

Figura 3. Variabilidade e tendência na concentração de $\mathrm{CH}_{4}$ e precipitação na atmosfera ao longo da série histórica. A variação interanual de anomalia de $\mathrm{CH}_{4}$ e precipitação pode ser observada na escala à direita em cinza. Na escala à esquerda na cor preta à anomalia acumulada, observa-se a tendência de acréscimo e decréscimo de concentração de $\mathrm{CH}_{4}$ e precipitação ao longo da série. Análises para os níveis $359 \mathrm{mb}$ (C), $260 \mathrm{mb}$ (B) e $160 \mathrm{mb}$ (A) de pressão atmosférica e precipitação (D).

Essa variabilidade interanual da concentração de $\mathrm{CH}_{4}$ (Figura 3A) pode ser explicada pela variabilidade interanual de precipitação (Figura 3D), com aumento das anomalias de precipitação e consequente aumento das áreas alagadas. Conforme Bousquet et al. (2006), áreas úmidas tem papel dominante na emissão de $\mathrm{CH}_{4}$ para atmosfera, variando em torno de ( $\pm 12 \mathrm{Tg} \mathrm{CH}_{4}$ ano $^{-1}$ ) explicando $70 \%$ das anomalias globais durante as duas últimas décadas. A variabilidade do $\mathrm{CH}_{4}$ em escala regional, como é o caso, está em concordância com a precipitação acumulada, sendo possível afirmar que existe uma correlação negativa entre as mesmas, justificada pelo pico máximo de precipitação coincidir com o mínimo de concentração de $\mathrm{CH}_{4}$, comparadas no mesmo período 2002-2013 e mesma resolução temporal de 8 dias.

A análise da série temporal da anomalia de concentração de $\mathrm{CH}_{4}$ com relação à anomalia de precipitação a partir de correlação cruzada determinou o tempo de defasagem entre as séries a 
fim de estabelecer o grau de associação entre estas (Figura 4). Verifica-se que para uma defasagem igual a zero a correlação é negativa, mostrando de forma geral que um aumento na altura precipitada reduz a emissão de $\mathrm{CH}_{4}$. Por outro lado, observam-se coeficientes de defasagem positivos em média 24 semanas depois dos picos negativos de defasagens, onde há as alturas maiores de precipitação. Isso é indicativo de que a diminuição da precipitação no Pantanal Mato-grossense influencia no aumento da emissão de $\mathrm{CH}_{4}$ para a atmosfera após aproximadamente 24 semanas, acompanhado por um decréscimo subsequente. Em medidas de superfície, a emissão de $\mathrm{CH}_{4}$ é maior no período chuvoso (Alvalá e Kirchhoff, 2000; Marani e Avalá, 2006; Da Silva et al., 2016). Contudo, existe um certo atraso entre o pico de chuva e pico de emissão de metano (Marani e Avalá, 2007) devido ao intervalo de tempo necessário para os processos de aumento do nível da lâmina d'água e aumento da metabolização anaeróbica pelas bactérias (metanogênicas) (Da Silva et al., 2016). Além disso, existe um intervalo entre os picos de emissão de superfície e o aumento da concentração na alta atmosfera (Ribeiro et al., 2016).

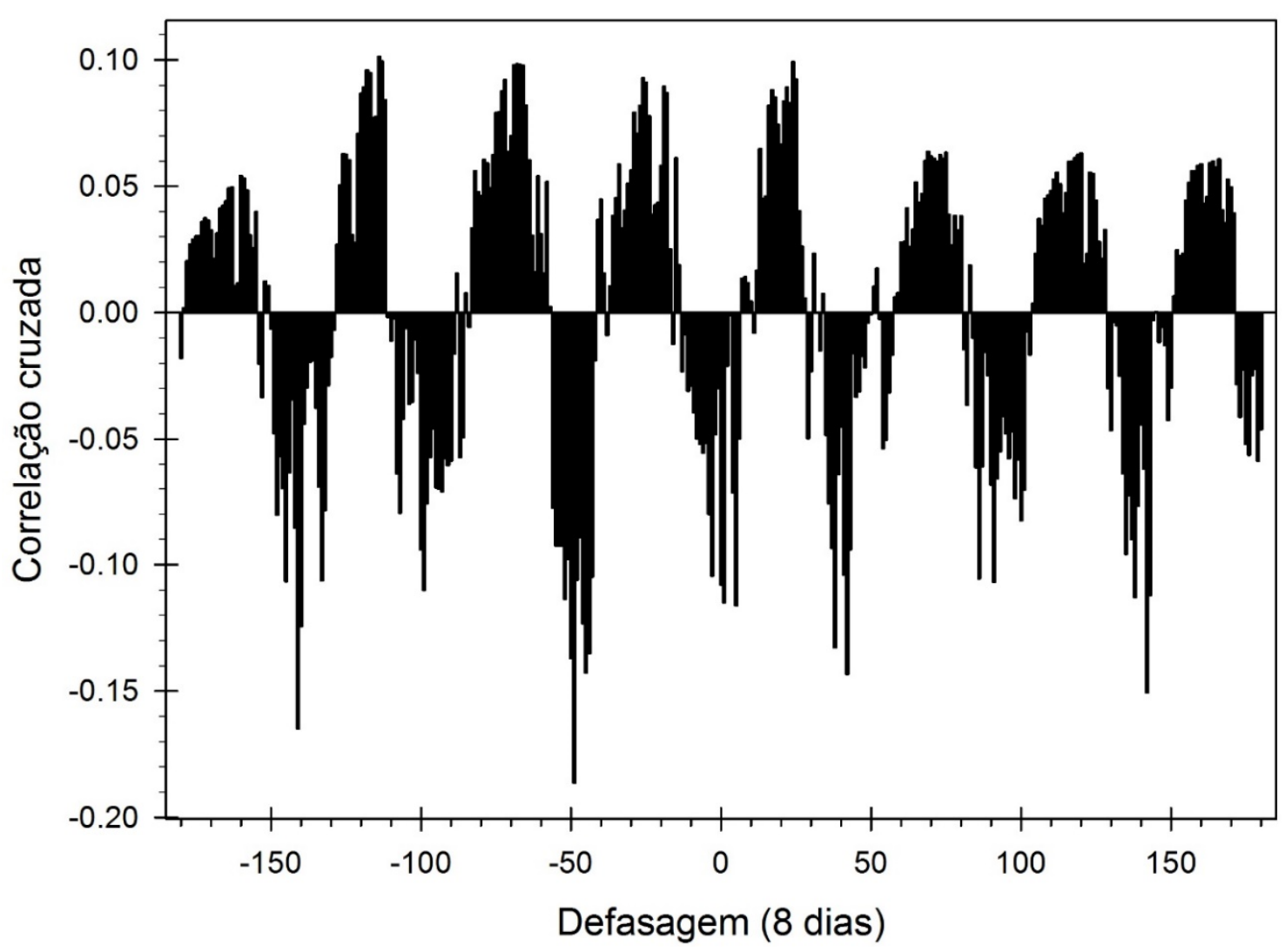

Figura 4. Correlação cruzada entre a série temporal de anomalia de precipitação e anomalia concentração do $\mathrm{CH}_{4}$ do período de 2002-2013. A escala defasagem representa o tempo de resposta do $\mathrm{CH}_{4}$ em relação à variação da precipitação.

Resultados de emissões pontuais e curto intervalo de tempo são reportados por vários estudos sendo incapazes de mostrar a dinâmica das emissões. Bastviken et al. (2010), mediram o fluxo de $\mathrm{CH}_{4}$ em 16 lagos na região do Pantanal Mato-grossense, realizando uma extrapolação utilizando a média de área alagada $\left(38880 \mathrm{~km}^{2}\right)$. O Pantanal produz um fluxo de 1,9 $\mathrm{Tg} \mathrm{CH} 4$ ano $^{-1}$. Estes resultados podem ser comparados aos de Marani e Alvalá (2006), que analisaram locais próximos ao rio Miranda também na região do Pantanal Mato-grossense, e ao utilizarem uma média maior de área alagada, obtiveram o valor de $3,3 \mathrm{Tg} \mathrm{CH}_{4}$ ano $^{-1}$, que representa 3,3\% da contribuição global de áreas alagadas. Os fluxos difusivos são 
correlacionados principalmente com características físico-químicas relacionadas ao aumento de matéria orgânica durante o alagamento no Pantanal (Da Silva et al., 2016). Áreas alagadas que compreendem florestas, ou que estão próximas a rios no Pantanal, tem altas taxas de produtividade bruta primaria, alta temperaturas e elevada radiação solar, resultando no aumento da taxa de decomposição, que por sua vez contribui no acréscimo de fluxo de $\mathrm{CH}_{4}$ para atmosfera (Bastviken et al., 2010).

$\mathrm{Na}$ análise da transformada ondoleta é possível identificar pela Figura 5A dois ciclos com intensidades de energia predominantes ao longo da série temporal de concentração de $\mathrm{CH}_{4} \mathrm{em}$ nível de pressão $260 \mathrm{mb}$ (2002-2013). Assim observa-se pela faixa da escala de cor no espectro de potência da Figura 5A que ocorreram ciclos na escala anual \pm 1 ano de 2004 a 2011 . O segundo ciclo foi interanual de \pm 2 anos de 2005 a 2011, com o conjunto de valores significativos delimitados pelo cone de influência. Este ciclo interanual de concentração de $\mathrm{CH}_{4}$ também foi observado por Marani e Alvalá (2006). Como também, ciclos aproximadamente anuais, mas de maior intensidade de energia (escala de 50 a 60 no espectro de potência ondoleta), ocorreram em período de 2006 a 2011. A intensidade de energia no período de 2006-2011 identificada na análise ondoleta, pode ser justificada pelo aumento na anomalia de precipitação.

Observa-se pelo espectro de potência global (Figura 5B), que os ciclos anual e interanual contêm as maiores variâncias, e além disso a variância do ciclo anual é maior e está, a maior parte, dentro do limite significativo.

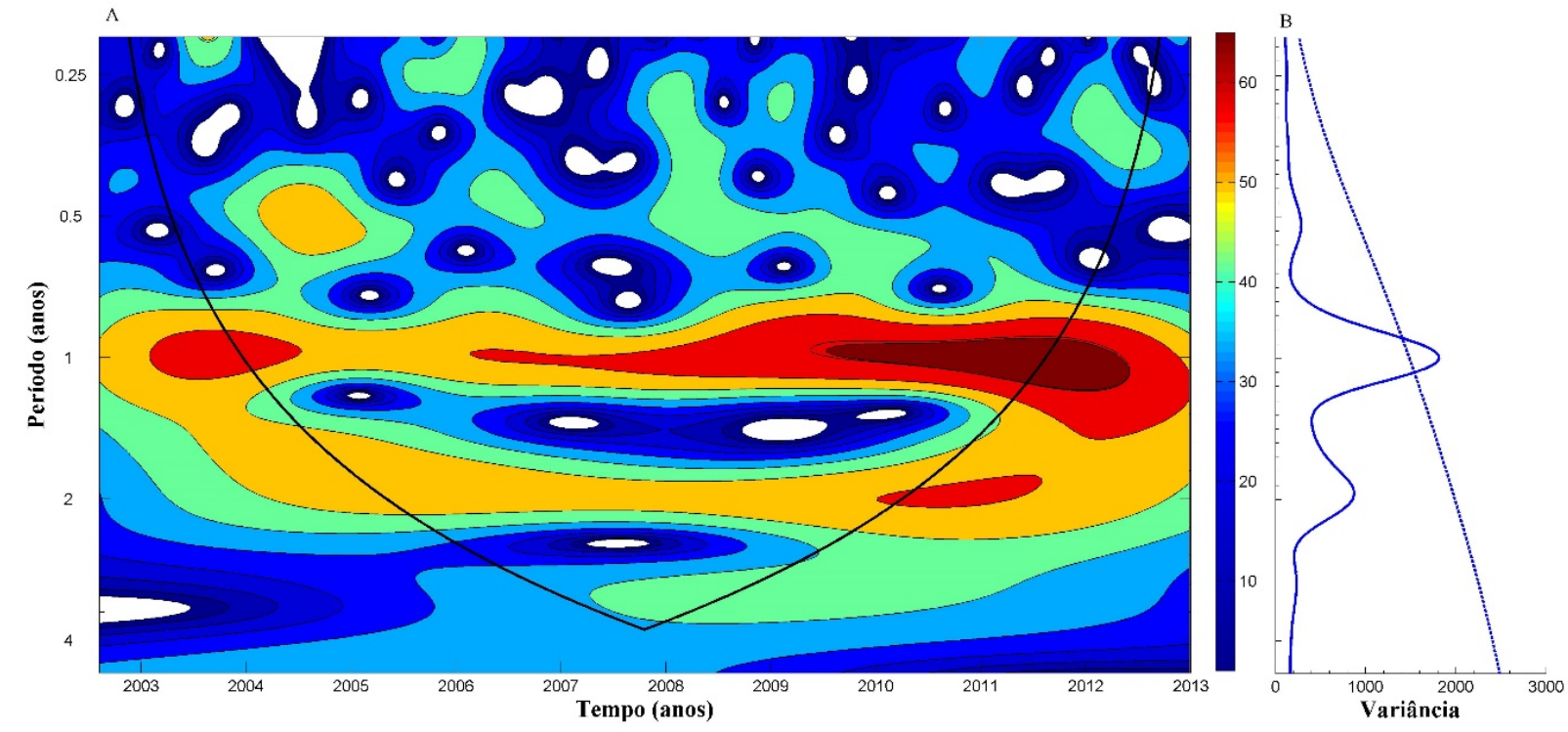

Figura 5. (A) Espectro de potência ondoleta. A região delimitada pela linha curva em forma de U representa o cone de influência (nível de 95\% de significância). (B) Espectro de potência global (linha azul). A linha pontilhada em azul é o nível de significância de $95 \%$ para o espectro global.

O resultado pode ser explicado pela variabilidade interanual observada pela anomalia da concentração de $\mathrm{CH}_{4}$. Portanto, a análise ondoleta reforça o resultado observado pela análise de anomalia de concentração de $\mathrm{CH}_{4}$. Houve variabilidade interanual e características cíclicas tanto em escala anual como interanual de concentração de $\mathrm{CH}_{4}$. $\mathrm{O}$ ciclo de maior predominância da periodicidade (acima de 60) foi aproximadamente 0,9 a 1 ano de ocorrência na segunda metade de 2009, em que esse ciclo variava entre a faixa 0,9 a 1,3 (Figura 5A) até meados de 2012; mas ele foi significativo até 2011 conforme delimitação da curva em forma de U (cone de influência na Figura 5A), justificado pela sazonalidade anual da precipitação no Pantanal. O cone de influência também indica que nenhum ciclo é significativo acima de 4 anos. Esses 
resultados são semelhante ao de Costa et al., (2014) e Ribeiro et al., (2016) com dados de sensoriamento relativos a áreas alagadas no Estado do Amazonas.

\section{CONCLUSÃO}

A investigação sobre o $\mathrm{CH}_{4}$ na atmosfera possibilitou analisar perturbações importantes na variabilidade da série temporal de concentração de $\mathrm{CH}_{4}$ sobre a região do Pantanal Mato-grossense de 2003-2013. Primeiramente observou-se um padrão sazonal da concentração de $\mathrm{CH}_{4}$ consistente com a sazonalidade da precipitação. Em escala anual, uma maior altura precipitada ocasionou maior concentração de $\mathrm{CH}_{4}$. Entretanto, em escala semanal observou-se pela análise de correlação cruzada que existe correlação negativa entre anomalia de chuva e concentração de $\mathrm{CH}_{4}$ na atmosfera com defasagem de 24 semanas, ocorrendo maior concentração de $\mathrm{CH}_{4}$ durante a estação seca. Esta defasagem corresponde ao intervalo de tempo necessário para a ampliação de área alagada como condição favorável a uma maior quantidade de decomposição de biomassa, que ocasiona emissões de $\mathrm{CH}_{4}$ para a atmosfera durante a estação subsequente. Ela também pode estar relacionada a um consumo de $\mathrm{CH}_{4}$ pelo radical $\mathrm{OH}^{-}$, que é seu principal sumidouro na atmosfera no período chuvoso.

Detectou-se variabilidade interanual de $\mathrm{CH}_{4}$ ao longo do período de 2002-2013, com aumento das anomalias positivas a partir de 2007, possivelmente relacionada ao acréscimo de anomalias positivas de precipitação a partir de 2006. Na análise ondoleta, observou-se ciclos anuais que ocorrem ao longo de quase toda série de temporal, variando entre 0,9 a 1,3 anos intensificando a partir de 2009 até 2013, indicando estar relacionado com a sazonalidade da precipitação e com um incremento da concentração de $\mathrm{CH}_{4}$. Contudo, houve ciclos interanuais variando aproximadamente de 1,5 a 2 anos.

Análises da concentração de $\mathrm{CH}_{4}$ sobre o Pantanal fornecem contribuições importantes sobre a dinâmica do $\mathrm{CH}_{4}$ na média e alta troposfera. Essas informações podem dar suporte a gestão de hídricos, e ainda ajudar no conhecimento de futuras mudanças climáticas e delinear estratégias de mitigação.

\section{REFERENCIAS}

ALVALÁ, P. C.; BOIAN, C.; KIRCHHOFF, V. W. J. H. Measurements of $\mathrm{CH}_{4}$ and $\mathrm{CO}_{2}$ during ship cruises in the South Atlantic. Atmospheric Environment, v. 38, p. 4583-4588, 2004. http://dx.doi.org/10.1016/j.atmosenv.2004.03.042

ALVALÁ, P. C.; KIRCHHOFF, V. W. J. H. Methane fluxes from the Pantanal floodplain in Brazil: seasonal variation. In: VAN HAM, J.; BAEDE, A. P. M.; MEYER, L. A.; YBEMA, R. (Eds.). Non-CO2 Greenhouse Gases: Scientific Understanding, Control and Implementation. Netherlands: Springer, 2000. p. 95-99. http://dx.doi.org/10.1007/978-94-015-9343-4_6

BASTVIKEN, D.; SANTORO, A. L.; MAROTTA, H.; PINHO, L. Q.; CALHEIROS, D. F.; CRILL, P. Methane emissions from Pantanal, South America, during the low water season: toward more comprehensive sampling. Environmental Science and Technology, v. 44, n. 14, p. 5450-5455, 2010. http://dx.doi.org/10.1021/es1005048

BEECHAM, S.; CHOWDHURY, R. K. Temporal characteristics and variability of point rainfall: a statistical and Wavelet analysis. International Journal of Climatology, v.30, p. 458-473, 2010. http://dx.doi.org/10.1002/joc.1901 
BOUSQUET, P.; CIAIS, P.; MILLER, J. B.; DLUGOKENCKY, E. J.; HAUGLUSTAINE, D. A.; PRIGENT, C. et al. Contribution of anthropogenic and natural sources to atmospheric methane variability. Nature, v.443, p. 439-443, 2006.

http://dx.doi.org/10.1038/nature05132

COSTA, P. S.; SIlVA, J. V.; SOUZA, R. A. F.; SOUZA, R. V. A.; JÚNIOR, N. P. F.; CARTAXO, E. F. Monitoring of Methane on Dams of hydroelectrics of the Amazon Basin from the Aqua Satellite information. Journal of Hyperspectral Remote Sensing, v.4, p. 19-30, 2013.

DA SILVA, M. G.; ALVALÁ, P. C.; MARANI, L.; FERREIRA, W. J. Análise da influência de parâmetros ambientais no fluxo de metano em áreas alagáveis e lagoas permanentes na região do Rio Abobral, no Pantanal, Brasil. Revista Ambiente \& Água, v. 11, n. 2, p. 327-338, 2016. http://dx.doi.org/10.4136/ambi-agua.1775

DLUGOKENCKY, E. J.; NISBET, E. G.; FISHER, R.; LOWRY, D. Global atmospheric methane: budget, changes and dangers. Royal Society Publishing, v.369, p.2058-2072, 2011. http://dx.doi.org/10.1098/rsta.2010.0341

HAMILTON, S. K.; SIPPEL, S. J.; MELACK, J. M. Comparison of inundation patterns among major South American floodplains. Journal of Geophysical Research, v.107, p. 1-14, 2002. http://dx.doi.org/10.1029/2000jd000306

KOEBSCH, F.; JURASINSKI, G.; KOCH, M.; HOFMANN, J. Controls for multi-scale temporal variation in ecosystem methane exchange during the growing season of a permanently inundated fen. Agricultural and Forest Meteorology, v.204 p. 94-105, 2015. http://dx.doi.org/10.1016/j.agrformet.2015.02.002

MARANI, L.; ALVALA, P. C. Methane emissions from lakes and floodplains in Pantanal, Brazil. Atmospheric Environment, v.41, p.1627-1633, 2006.

http://dx.doi.org/10.1016/j.atmosenv.2006.10.046

MELTON, J. R.; WANIA, R.; HODSON, E. L.; POULTER, B.; RINGEVAL, B.; SPAHNI, R. et al. Present state of global wetland extent and wetland methane modelling: conclusions from a model intercomparison project (WETCHIMP). Biogeosciences, v. 10, p. 753-788, 2013. http://hdl.handle.net/11858/00-001M-0000-000F-ED12-B

MCNORTON, J.; CHIPPERFIELD, M. P.; GLOOR, M.; WILSON, C.; FENG, W.; HAYMAN, G. D. et al. Role of $\mathrm{OH}$ variability in the stalling of the global atmospheric $\mathrm{CH}_{4}$ growth rate from 1999 to 2006. Atmospheric Chemistry and Physics, p. 1-24, 2016. http://dx.doi.org/10.5194/acp-2015-1029

PENATTI, N. C.; RIBEIRO, A. T. I.; FERREIRA, L. G.; ARANTES, A. E.; COE, M. T. Satellite-based hydrological dynamics of the world's largest continuous wetland. Remote Sensing of Environment, p. 1-13, 2015. http://dx.doi.org/10.1016/j.rse.2015.08.031

RIBEIRO, I. O.; DE SOUZA, R. A. F.; ANDREOLI, R. V.; KAYANO, M. T.; DOS SANTOS COSTA, P. Spatiotemporal variability of methane over the Amazon from satellite observations. Advances in Atmospheric Sciences, v. 33, n. 7, p. 852-864, 2016. http://dx.doi.org/10.1007/s00376-016-5138-7 
SPIVAKOVSKY, C. M.; YEVICH, R.; LOGAN, J. A.; WOFSY, S. C.; MCELROY, M. B.; PRATHER, M. J. Tropospheric $\mathrm{OH}$ in a three-dimensional chemical tracer model: An assessment based on observations of $\mathrm{CH} 3 \mathrm{CCl} 3$. Journal of Geophysical Research: Atmospheres, v. 95, n. D11, p. 18441-18471, 1990. http://dx.doi.org/10.1029/JD095iD11p18441

TORRENCE, C.; COMPO, G. P. A Practical Guide to Wavelet Analysis. Bulletin of the American Meteorological Society, v.79, p. 61-78, 1997. http://dx.doi.org/10.1175/1520-0477(1998)079\%3C0061:APGTWA\%3E2.0.CO;2

VOURLITIS, G. L.; LOBO, F. A.; LAWRENCE, S.; LUCENA, I. C; PINTO Jr., O. B.; DALMAGRO, H. J.; et al. Variations in Stand Structure and Diversity along a Soil Fertility Gradient in a Brazilian Savanna (Cerrado) in Southern Mato Grosso. Forest, Range \& Wildland Soils, p. 1370-1379, 2013. http://dx.doi.org/10.2136/sssaj2012.0336

XIONG, X.; BARNET, C.; MADDY, E.; WOFSY, S. C.; CHEN, L.; KARION, A. et al. Detection of methane depletion associated with stratospheric intrusion by atmospheric infrared sounder (AIRS), Geophysical Research Letters, v.40, p. 2455-2459, 2013. http://dx.doi.org/10.1002/grl.50476 